PREPARED FOR THE U.S. DEPARTMENT OF ENERGY, UNDER CONTRACT DE-AC02-76CH03073

PPPL-3484

PPPL-3484

UC-70

Expulsion of Energetic lons from the Plasma Core in the Presence of Sawtooth Oscillations in Spherical Tokamaks

by

Ya. I. Kolesnichenko, V.V. Lutsenko,

R.B. White, and Yu. V. Yakovenko

August 2000

$=$ PPPL

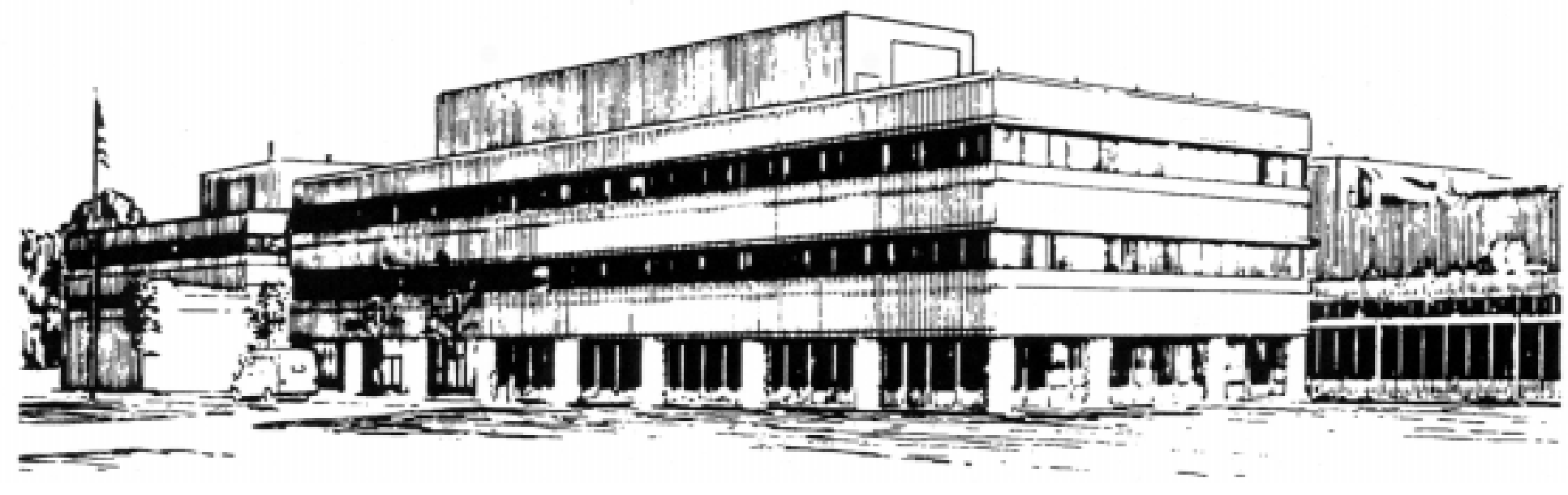

PRINCETON PLASMA PHYSICS LABORATORY PRINCETON UNIVERSITY, PRINCETON, NEW JERSEY 


\section{PPPL Reports Disclaimer}

This report was prepared as an account of work sponsored by an agency of the United States Government. Neither the United States Government nor any agency thereof, nor any of their employees, makes any warranty, express or implied, or assumes any legal liability or responsibility for the accuracy, completeness, or usefulness of any information, apparatus, product, or process disclosed, or represents that its use would not infringe privately owned rights. Reference herein to any specific commercial product, process, or service by trade name, trademark, manufacturer, or otherwise, does not necessarily constitute or imply its endorsement, recommendation, or favoring by the United States Government or any agency thereof. The views and opinions of authors expressed herein do not necessarily state or reflect those of the United States Government or any agency thereof.

\section{Availability}

This report is posted on the U.S. Department of Energy's Princeton Plasma Physics Laboratory Publications and Reports web site in Calendar Year 2000. The home page for PPPL Reports and Publications is: http://www.pppl.gov/pub_report/

DOE and DOE Contractors can obtain copies of this report from:

U.S. Department of Energy

Office of Scientific and Technical Information

DOE Technical Information Services (DTIS)

P.O. Box 62

Oak Ridge, TN 37831

Telephone: (865) 576-8401

Fax: (865) 576-5728

Email: reports@adonis.osti.gov

This report is available to the general public from:

National Technical Information Service

U.S. Department of Commerce

5285 Port Royal Road

Springfield, VA 22161

Telephone: $1-800-553-6847$ or

(703) $605-6000$

Fax: (703) 321-8547

Internet: http://www.ntis.gov/ordering.htm 


\title{
Expulsion of Energetic Ions from the Plasma Core in the Presence of Sawtooth Oscillations in Spherical Tokamaks
}

\author{
Ya.I. KOLESNICHENKO ${ }^{1}$, V.V. LUTSENKO ${ }^{1}$, \\ R.B. WHITE ${ }^{2}$, Yu.V. YAKOVENKO ${ }^{1}$ \\ ${ }^{1}$ Scientific Centre "Institute for Nuclear Research", Kyiv, 03680, Ukraine \\ 2 Princeton Plasma Physics Laboratory, P.O. Box 451, Princeton, NJ, 08543, USA
}

\begin{abstract}
It is shown that high-beta-induced changes in the wave-particle interaction can strongly influence the fast ion transport during sawtooth oscillations.
\end{abstract}

\section{INTRODUCTION}

The ratio of the plasma pressure to the magnetic field pressure $(\beta)$ in spherical tokamaks (ST) can essentially exceed that in conventional tokamaks (CT). This fact leads to differences in the physical processes in STs and CTs. In particular, as was shown recently, when $\beta$ is sufficiently high, the fishbone mode associated with the trapped particles is stable [1]. In the present work, we consider the transport of the energetic ions in a high- $\beta$ plasma with sawtooth oscillations.

It was predicted theoretically [2] and confirmed experimentally [3] that the energetic ion transport in CTs with sawtooth oscillations depends on the particle energy and pitch angle (see also a recent invited paper [4]). It was found that there exists a critical energy, $\mathcal{E}_{\text {crit }}$, such that the well-trapped ions with the energy higher than $\mathcal{E}_{\text {crit }}$ are not sensitive to the sawtooth crash, whereas the ions with $\mathcal{E}<\mathcal{E}_{\text {crit }}$ are strongly redistributed by the crash. The critical energy is given by $[2,4]$

$$
\mathcal{E}_{\text {crit }}=\left.\frac{2 \pi M k r R_{0} \omega_{B}}{\tau_{c r}}\right|_{r=r_{s}} \propto \frac{A_{s} B S}{\tau_{c r}},
$$


where $\tau_{c r}$ is the crash duration, $k$ is the ellipticity of the plasma cross section, $r_{s}$ is the radius of the $q=1$ flux surface, $M$ is the ion mass, $\omega_{B}$ is the cyclotron frequency, $R_{0}$ is the large radius of the torus, $S$ is the square of the $q=1$ flux-surface cross-section, $A_{s}=R_{0} / r_{s}$, and $B$ is the magnetic field strength. When $\tau_{c r} \sim 10^{-4} \mathrm{~s}, \mathcal{E}_{\text {crit }}$ varies in the range $300 \div 700 \mathrm{keV}$ for $\alpha$-particles in TFTR and JET, depending on plasma parameters. Because the product $A_{s} B S$ in STs is relatively small, one can conclude from Eq. (1) that $\mathcal{E}_{\text {crit }}$ in STs is much less than that in CTs and, thus, the sawtooth crashes in STs will either not affect the trapped energetic ions or affect them selectively through resonance effects $[4,5]$. However, the existing theory is relevant to plasmas with sufficiently small $\beta$ when the effects of plasma diamagnetism are negligible. This motivated the investigation reported in the present work aimed at clarifying the physical mechanisms responsible for the sawtooth-induced transport of the trapped energetic ions in high- $\beta$ discharges of STs.

\section{PARTICLE MOTION IN A HIGH- $\beta$ PLASMA DURING THE SAWTOOTH CRASH}

We consider an MHD-active plasma with the MHD activity associated with the development of the $m=n=1$ helical perturbation accompanied by the reconnection of the magnetic field lines in a narrow current layer, which is presumably the case during the sawtooth crashes. We assume that the helical symmetry is conserved during the MHD events, so that the magnetic flux surfaces can be described by the helical flux function $\psi(r, \alpha, t)$, where $r$ is the radial coordinate such that $r=$ const describes the flux surfaces before and after the crash; $\alpha \equiv \theta-\phi, \theta$, and $\phi$ are the helical, poloidal, and toroidal angles, respectively; $t$ is time. In addition, we assume that the duration of the sawtooth crashes (or other similar MHD events) well exceeds the bounce period of the trapped energetic ions and that $\Delta r_{b} \ll r_{m i x}$, where $\Delta r_{b}$ is the banana width, and $r_{m i x}$ is the sawtooth mixing radius.

Using these assumptions, we have derived the following equations of the particle bounce averaged motion: 


$$
\begin{gathered}
\dot{r}=v_{E}^{1}+\left\langle v_{\|}^{1}\right\rangle+\frac{\mu \bar{B}}{M k \omega_{B} R_{0}}\left(G-\frac{1}{\epsilon}\right) \frac{\partial \bar{B}}{\partial \alpha}, \\
\dot{\alpha}=v_{E}^{2}+\left\langle v_{\|}^{2}\right\rangle-\frac{\mu \bar{B}}{M k \omega_{B} R_{0}}\left[\left(G-\frac{1}{\epsilon}\right) \frac{\partial \bar{B}}{\partial r}+\frac{G}{r}\right],
\end{gathered}
$$

where $\vec{v}_{E}$ is the velocity of the $\vec{E} \times \vec{B}$ drift, $\vec{E}$ being the electric field associated with the evolution of the magnetic configuration and concomitant plasma motion; $\vec{v}_{\|}$is the particle velocity along the magnetic field; the brackets denote bounce averaging; the superscripts mean the contravariant vector components; $\bar{B}=B_{0} \sqrt{1-8 \pi p(r, \alpha) / B_{0}^{2}}, p$ is the plasma pressure, $B_{0}$ is the vacuum magnetic field at the magnetic axis, $\mu$ is the particle magnetic moment, $R=R_{0}(1+\epsilon \cos \theta), \epsilon=r / R_{0}, G=2 \mathbf{E}(\kappa) / \mathbf{K}(\kappa)-1, \mathbf{K}(\kappa)$ and $\mathbf{E}(\kappa)$ are the complete elliptic integrals of the first and second kind, respectively; $\kappa$ is the particle trapping parameter which depends on $\bar{B}$, and thus, on the plasma pressure as follows:

$$
\kappa^{2}(r, \alpha)=\frac{\mathcal{E}(r, \alpha)-\mu \bar{B}(r, \alpha)(1-\epsilon)}{2 \mu \bar{B}(r, \alpha) \epsilon} .
$$

Let us analyse Eqs. (2), (3). The first terms in these equations describe the $\vec{E} \times \vec{B}$ drift. These are the terms driving the particle redistribution by making the particles frozen into the evolving flux surfaces [2]. The motion along the field lines described by the second terms also attaches particles to the flux surfaces, but its effect is negligible for the narrow-orbit trapped particles. The conventional precession is described by the last term in Eq. (3). It competes with the $\vec{E} \times \vec{B}$ drift motion resulting in $\mathcal{E}_{\text {crit }}$ (the latter is determined from the condition $\tau_{p r}=\tau_{c r}$, where $\tau_{p r}$ is the characteristic precession time). The other terms in Eqs. (2), (3) are associated with the plasma diamagnetism. Taking into account that $\bar{B}=\bar{B}(p)$ and $p=p(\psi)$, we conclude from Eqs (2), (3) that the precession associated with the plasma diamagnetism conserves $\psi$, i.e., it leads to the particle motion along the flux surfaces rather than along the $r=$ const surfaces.

Thus, the conventional precession is the only factor preventing the particle redistribution by the crash. The diamagnetic precession overrides the conventional precession and, thus, attaches the particles to the moving flux surfaces when $\partial \ln \bar{B} / \partial \ln r>\epsilon$, which leads to the estimate $\beta\left(r_{s}\right)>\epsilon\left(r_{s}\right)$, where $\beta\left(r_{s}\right) \equiv 8 \pi p\left(r_{s}\right) / B_{0}^{2}$. The latter is essentially 
the condition of the existence of the magnetic valley, i.e., a region in the vicinity of the magnetic axis where the equilibrium magnetic field strength has a minimum.

It is of interest to carry out a numerical simulation of the particle transport during a sawtooth crash. With this purpose we use a set of equations consisting of Eqs. (2), (3), (with $v_{E}^{1}=-c\left(B_{0} r k\right)^{-1}(\partial \Phi / \partial \alpha), v_{E}^{2}=c\left(B_{0} r k\right)^{-1}(\partial \Phi / \partial r), \Phi$ is the potential of the crash-induced electric field, and $\left\langle v_{\|}^{1}\right\rangle=-\left(B_{0} r k\right)^{-1}\left\langle v_{\|} / R\right\rangle \partial \psi / \partial \alpha,\left\langle v_{\|}^{2}\right\rangle=$ $\left.\left(B_{0} r k\right)^{-1}\left\langle v_{\|} / R\right\rangle \partial \psi / \partial r\right)$, the equation $J(r, \alpha, \kappa) \equiv \oint v_{\|} d l=$ const (which determines the time dependence of $\kappa$ ), the equation $\vec{E} \cdot \vec{B}=0$ (which connects $\Phi$ and $\psi$ ), and the equations for $\psi(r, \alpha, \phi, t)$ from the crash model of Ref. [2] approximating the Kadomtsev type of the crash. The results of calculations for a well-trapped particle with $\mathcal{E} \gg \mathcal{E}_{\text {crit }}$ are shown in Fig. 1. We observe that the increase of $\beta$ strongly changes the effect of the sawtooth crash on the particle. Namely, the particle, which was not sensitive to the crash at low $\beta$, is expelled from the plasma core in the high- $\beta$ plasma.

\section{CONCLUSIONS}

Our analysis has shown that the diamagnetism leads to the precessional motion along the disturbed flux surfaces. Therefore, this is a factor promoting the particle redistribution during the sawtooth crashes. It is found that the effect of the diamagnetism dominates in the precessional motion when the plasma pressure gradient is sufficiently large to result in the "valley" in the equilibrium magnetic field. In this case, the well trapped ions move together with the evolving flux surfaces even when their energy exceeds $\mathcal{E}_{\text {crit }}$.

The finite orbit width effects (resonance phenomena, stochasticity) may determine the transport of the various groups of the energetic ions in the MHD active plasmas of STs. They should be studied in future.

In conclusion, we have shown for the first time that when the particle energy exceeds a certain critical magnitude, the influence of sawtooth oscillations (or other similar forms of the plasma MHD activity) on the transport of the energetic ions in high- $\beta$ discharges of STs essentially differs from that in CTs. Namely, in STs, in contrast to CTs, trapped 
particles with $\mathcal{E}>\mathcal{E}_{\text {crit }}$ and peaked radial distribution are expelled from the plasma core because of the interaction with MHD perturbations.

\section{ACKNOWLEDGEMENTS}

The research described in this work was made possible in part by the IAEA Research Contract No. 10539 and Award No. UP2-2114 of the U.S. Civilian Research \& Development Foundation.

\section{REFERENCES}

[1] Ya.I. Kolesnichenko, V.V. Lutsenko, V.S. Marchenko, Phys. Rev. Lett. 82 (1999) 3260.

[2] Ya.I. Kolesnichenko, Yu.V. Yakovenko, in Collection of papers presented at the IV IAEA Tech. Com. Mtg. on Alpha Particles in Fusion Research (Princeton, April 1995); Nucl. Fusion 36 (1996) 159.

[3] N.N. Gorelenkov, R.V. Budny, H.H. Duong, et al., Nucl. Fusion 37 (1997) 1053.

[4] Ya.I. Kolesnichenko, V.V. Lutsenko, R.B. White, Yu.V. Yakovenko, "Effect of sawtooth oscillations on energetic ions", Invited talk at 6th IAEA TC Mtg on Energetic Particles in Magnetic Confinement Systems, Naka, Japan (1999); Report PPPL-3404 (1999); Nucl. Fusion 40 (2000) 1325.

[5] Ya.I. Kolesnichenko, V.V. Lutsenko, R.B. White, Yu.V. Yakovenko, Phys. Rev. Lett. $84(2000) 2152$. 


\section{FIGURES}
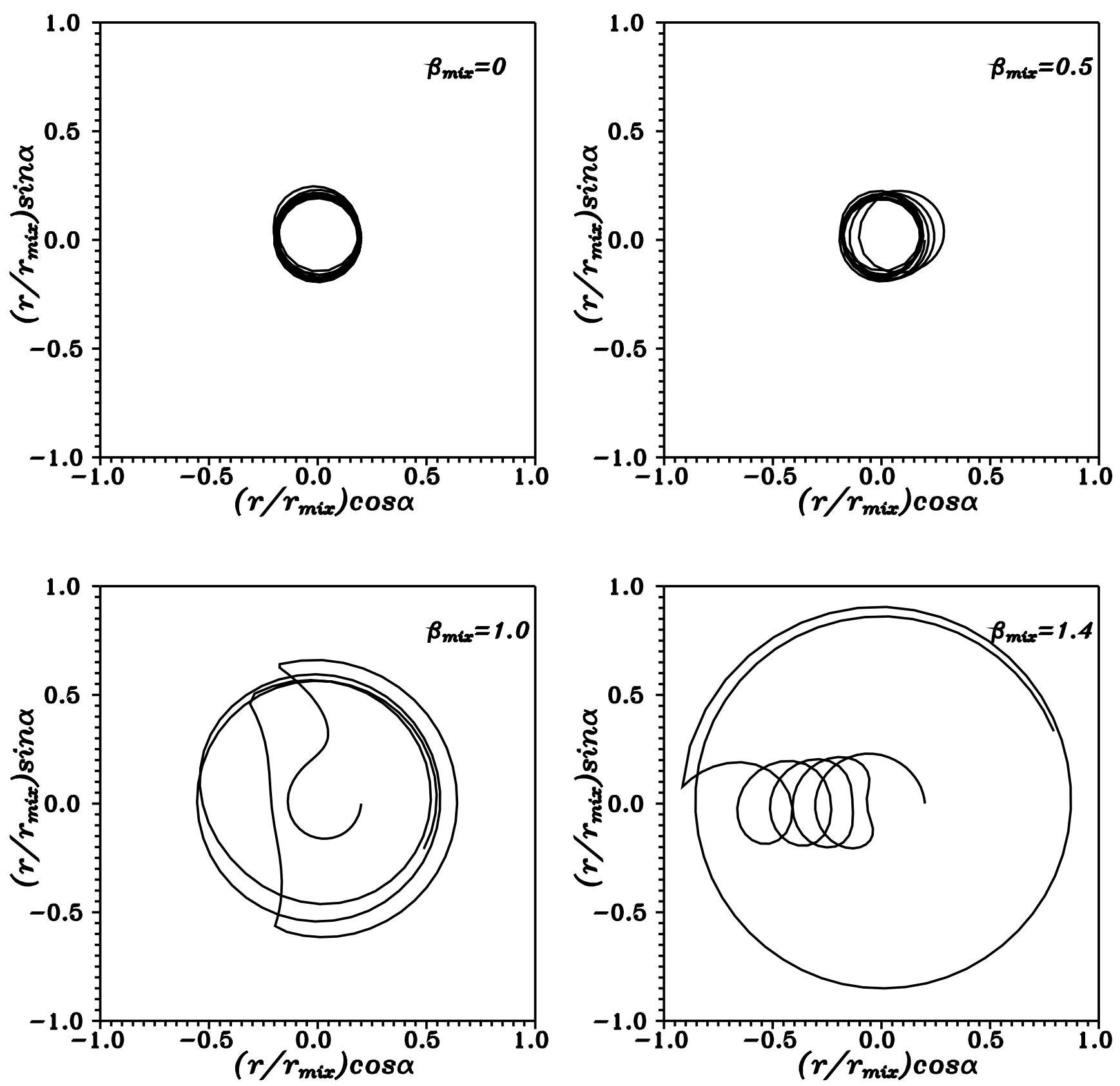

FIG. 1. Motion of a well-trapped energetic ion with $\mathcal{E}>\mathcal{E}_{\text {crit }}$ in plasmas with various $\beta$ during sawtooth crashes. We observe that the crash has no influence on the ion when $\beta=0$, whereas the ion is strongly displaced when $\beta$ is high. 
The Princeton Plasma Physics Laboratory is operated by Princeton University under contract with the U.S. Department of Energy.

\author{
Information Services \\ Princeton Plasma Physics Laboratory \\ P.O. Box 451 \\ Princeton, NJ 08543
}

Phone: 609-243-2750

Fax: 609-243-2751

e-mail: pppl_info@pppl.gov

Internet Address: http://www.pppl.gov 